\title{
Quantitating the Relative Abundance of Isoaspartyl Residues in Deamidated Proteins by Electron Capture Dissociation
}

\author{
Jason J. Cournoyer* \\ Department of Chemistry, Boston University, Boston, Massachusetts, USA
}

\author{
Cheng Lin, Michael J. Bowman, and Peter B. O'Connor \\ Department of Biochemistry, Mass Spectrometry Resource, Boston University School of Medicine, Boston, \\ Massachusetts, USA
}

\begin{abstract}
Relative quantitation of aspartyl and isoaspartyl residue mixtures from asparagine deamidation is demonstrated using electron capture dissociation without prior HPLC separation. The method utilizes the linear relationship found between the relative abundance of the isoaspartyl diagnostic ion, $z_{n}-57$, and \% isoaspartyl content based on the ECD spectra of known isoaspartyl/aspartyl mixtures of synthetic peptides. The observed linearity appears to be sequence independent because the relationship exists despite sequence variations and changes in backbone fragment abundances when isoaspartyl and aspartyl residues are interchanged. Furthermore, a new method to calculate the relative abundances of isomer from protein deamidation without synthetic peptides is proposed and tested using a linear peptide released by protein digestion that contains the deamidation site. The proteolytic peptide can be rapidly aged to the expected 3:1 (isoaspartyl:aspartyl) mixture to generate a two-point calibration standard for ECD analysis. The procedure can then be used to determine the relative abundance of deamidation products from in vivo or in vitro protein aging experiments. (J Am Soc Mass Spectrom 2007, 18, 48-56) @ 2007 American Society for Mass Spectrometry
\end{abstract}

$\mathrm{I}$ $n$ peptides and proteins, deamidation of asparaginyl and isomerization of aspartyl (Asp) residues can occur spontaneously to form a mixture of isoaspartyl (isoAsp) and Asp residues (Figure 1) [1-3], a process believed to be a common in vivo degenerative pathway for proteins that affects their structure [4-6] and activity [7-9]. The rate of deamidation is influenced by steric effects from the C-terminal amino acid neighbor [10] to asparagine and local three-dimensional structure [11]. For example, under physiological conditions, linear peptides with -NI- were shown to have a deamidation half-life of $300 \mathrm{~d}$ opposed to peptides with -NG- that had a half-life of $1 \mathrm{~d}$ [10]. Therefore, deamidation of -NG- is a common peptide modification that results from in-gel digestion protocols that require overnight incubation in ammonium bicarbonate $(\sim \mathrm{pH} 8.5)$ with trypsin [12, 13]. Asp isomerization is also possible (Figure 1), but occurs $\sim 40$ times slower than deamidation [2] and is therefore a less prevalent modification.

Published online September 25, 2006

Address reprint requests to Professor P. B. O'Connor, Department of Biochemistry, Mass Spectrometry Resource, Boston University School of Medicine, 715 Albany Street, Boston, MA 02118, USA. E-mail: poconnor@bu.edu

* Also at Department of Biochemistry, Mass Spectrometry Resource, Boston University School of Medicine.
The isoAsp form has the $\beta$-carbon originally of the side chain inserted into the backbone altering the fundamental protein structure and is believed to contribute to disruption of protein structure and function more than the Asp form [14]. Hydrolysis of the succinimide intermediate in linear peptides typically results in an approximate 3:1 ratio of products favoring the isoAsp form [15-21], but ratios from succinimide hydrolysis within a structurally restricted environment, such as within a protein's tertiary structure, can deviate from this ratio [22]. For example, N67 of ribonuclease A ultimately deamidated to a 3:2 ratio in favor of the Asp form due to the restricted flexibility of the region by two flanking disulfide bonds [23]. Therefore, a method that determines the relative abundance of the products is necessary for complete characterization of asparaginyl deamidation and Asp isomerization that is suspected to affect a protein's structure and function.

Analytical techniques spearheading the analysis of asparaginyl deamidation include radiolabeling, isoelectric focusing (IEF) gel electrophoresis and ion-exchange high-performance liquid chromatography (HPLC), reverse phase HPLC, Edman degradation, and mass spectrometry (MS). Radioactive methods utilize the enzyme protein isoaspartyl methyl transferase (PIMT) to selectively methylate isoAsp residues in peptides and 


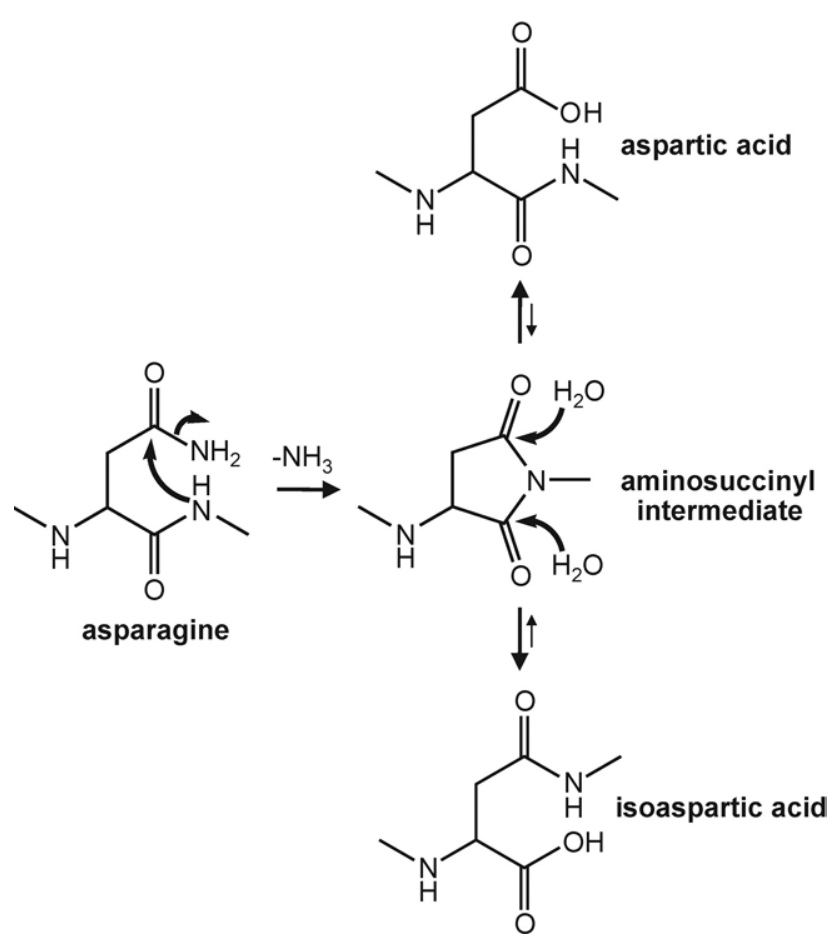

Figure 1. The standard model for deamidation of asparaginyl or isomerization of Asp residues to a mixture of Asp and isoAsp residues.

proteins with isotopically labeled methyl groups, thus detecting deamidation, but not localizing the site or determining the relative abundance of the products [24, 25]. Separation of proteins from their deamidated counterparts can be done by ion-exchange HPLC [23, 26, 27] or IEF gel electrophoresis $[14,28]$ because there is a net change in the isoelectric point with deamidation. However, the methods are not used for differentiation and relative quantitation of the deamidation products because the acidity constants of the isomers are so similar; isoAsp and Asp were shown to have a difference of only $0.7 \mathrm{pK}_{\mathrm{a}}$ units [17]. Deamidated proteins are often digested to localize the deamidation site and determine the ratio of products by reverse-phase HPLC $[19,20$, $26]$, but separation of the isomers can be challenging.

Several difficulties are encountered when separating Asp/isoAsp peptides by reverse-phase HPLC; customized separation for each peptide mixture, increased analysis time and sample consumption, and interference from other peptides. The slight structural differences between isoAsp and Asp residues in peptides can make their separation challenging, so shallow gradients and long run times are often necessary for baseline separation. For example, a gradient of $15-19 \%$ organic mobile phase in $60 \mathrm{~min}$ was required to achieve baseline separation of isoAsp/Asp peptides from a tryptic digest [20]. Furthermore, larger peptides reduce the influence of the structural difference (isoAsp versus Asp) that is critical for separation and may render these mixtures inseparable. Many HPLC runs are often required before adequate separation is achieved, thus increasing total analysis time and sample consumption. Finally, isomers existing in a complex mixture, such as a protein digest, may have retention times similar to other species making their relative quantitation more problematic.

Once separation is completed, Edman degradation or MS can differentiate the isomers. Edman degradation, which is blocked by isoAsp residues [29], requires both chemical and HPLC procedures for sequence determination, thus lengthening analysis time. Substituting Edman degradation for MS can shorten analysis time but differentiation is difficult because there is no mass difference between the isomers. Differences in the fragment mass spectra of the isomers are therefore required for differentiation.

Collisionally activated dissociation (CAD) $[30,31]$ in MS can differentiate Asp from isoAsp residues using fragmentation patterns particular to each form but peptide standards are necessary because the method relies on the relative intensity of fragments for differentiation [32-35]. Electron capture dissociation (ECD) [36] in a Fourier transform mass spectrometer (FTMS) $[20,37]$ or electron-transfer dissociation (ETD) [38] in an ion trap can differentiate the two forms using a diagnostic ion unique to the isoAsp form without the need for peptide standards.

ECD is a sequencing tool that involves the reaction of electrons with gas-phase multiply charged peptide and protein cations. The captured electron initiates $\mathrm{N}-\mathrm{C}_{\alpha}$ bond cleavage producing $\mathrm{c}$ and $\mathrm{z}$ - type fragment ions ( $\mathrm{N}$ - and C-terminal fragments, respectively) and can preserve posttranslational modifications linked to the fragments [39, 40]. ECD can also cleave bonds in amino acid side chains such as the $C_{\alpha}-C_{\beta}$ bond [41]. For Asp residues, this results in $\mathrm{M}^{+\cdot}-60$, but for isoAsp residues, cleavage of the $\mathrm{C}_{\alpha}-\mathrm{C}_{\beta}$ backbone bond by ECD generates $c_{1-n}+57$ and $z_{n}-57$ ( $n$ is isoAsp, 1 is length of the peptide) fragment ions thus detecting and localizing the isoAsp residue. Analysis of deamidated peptides from proteins with HPLC and ECD [20] demonstrated that the combined technique easily differentiates and determines the relative abundance of products but the method is still subject to the limitations associated with HPLC method development described above.

Relative quantitation of isomers with MS alone using fragmentation patterns unique to each form determined the relative abundances of sulfated disaccharides released from digestion of chondroitin [42, 43], suggesting that an analogous method could be developed for relative isoAsp/Asp quantitation. The possible sulfate positions in disaccharides from chondroitin are few compared with the possible amino acid sequences obtained from proteins so a fragmentation trend reproducible for all peptides from proteins is ideal. In this study, ECD spectra of three shynthetic peptides mixed at different percent isoAsp/Asp compositions showed a reproducible correlation between the relative abundance of the isoAsp diagnostic ion and mixture composition. A method is then suggested using this relationship for complete characterization of asparagine 
deamidation in a protein and demonstrated using cytochrome $c$ as a model.

\section{Experimental}

\section{Materials}

Rink resin (1.2 mmol/g loading), HBTU [O-(benzotriazol-1-yl)- $N, N, N \quad N^{\prime}$-tetramethyluronium hexafluorophosphate] and the FMOC [N- $\alpha$-(9-fluorenylmethoxycarbonyl)]-protected amino acids glutamic acid, glutamine, isoleucine, histidine, tyrosine, and threonine were purchased from Advanced Chemtech (Louisville, KY). FMOC-protected isoAsp was purchased from NovaBiochem (La Jolla, CA). Trypsin was purchased from Roche chemicals (Indianapolis, IN). All other chemicals were purchased from Sigma Aldrich (St. Louis, MO).

\section{Peptide Synthesis}

Peptides were synthesized by a standard manual solidphase peptide synthetic method in $1 \mathrm{~mL}$ polypropylene syringe barrels (Supelco, St. Louis, MO) utilizing a polyethylene frit $(0.2 \mathrm{~mL})$. For each step, $500 \mathrm{uL}$ of the appropriate wash or reaction solution was added to the tube, sealed on both ends with stoppers, mixed on a rotisserie shaker for the specified amount of time, and the remaining solution was removed by vacuum filtration (the terms " $\mathrm{X} \times \mathrm{Y}$ min)" means mixing for $\mathrm{Y}$ min followed by filtration, done $X$ times). In a typical reaction sequence: resin was pretreated with DMF (N,N-dimethylformamide) $(3 \times 1 \mathrm{~min})$; treated with $20 \%$ piperidine solution in DMF $(1 \times 1 \mathrm{~min}, 1 \times 20 \mathrm{~min})$ to remove the FMOC protecting group; washed to provide the free amine $[\mathrm{DMF}(3 \times 1 \mathrm{~min}), \mathrm{DCM}$ (dichloromethane) $(3 \times 1 \mathrm{~min})$, ethanol $(3 \times 1 \mathrm{~min})$, $\mathrm{DCM}(3 \times 1 \mathrm{~min})$, and DMF $(3 \times 1 \mathrm{~min})]$; reacted with a $600 \mathrm{~mL}$ mixture of the appropriate amino acid solution (two equivalents of FMOC-amino acid, HBTU and DIPEA ( $N$-ethyldiisopropylethanolamine) in DMF preactivated by mixing for 5-8 $\mathrm{min}$ ) for $2 \mathrm{~h}$; washed to remove residual reactants $[\mathrm{DMF}(3 \times 1 \mathrm{~min}), \mathrm{DCM}(3 \times$ $1 \mathrm{~min})$ ]; dried and stored under vacuum or the cycle was repeated for the next amino acid. About two residues per day were added making total synthesis time 3 weeks for three sets of unpurified peptides. When peptide synthesis was complete, pretreatment with DMF, deprotection and washing with ethanol was completed and the resin was dried under vacuum overnight. Cleavage of the peptide from the resin was done using a $1 \mathrm{~mL}$ solution of TFA (trifluoroacetic acid):DCM:TIPS (triisopropylsilane):water (45:45:5:5) with mixing for $2 \mathrm{~h}$ under nitrogen followed by precipitation in cold ether. All peptides were purified using reverse phase HPLC with single wavelength detection at $278 \mathrm{~nm}$ using a mobile phase system of 5\% acetonitrile in water with $0.05 \%$ TFA (mobile phase A) and $100 \%$ acetonitrile with $0.035 \%$ TFA (mobile phase B).
YWQHTA(isoD/D)QFR-NH $\mathrm{N}_{2}$ was purified by a gradient of $7-15 \%$ B in $30 \mathrm{~min}$ on a $4.6 \mathrm{~mm}$ i.d. $\times 250 \mathrm{~mm}$ C18 column (Vydac, Hesperia, CA) at $0.5 \mathrm{~mL} / \mathrm{min}$ using a System Gold 125 pump and 166 detector (Beckman Coulter, Fullerton, CA). Y(isoD/D)FIEYVR-NH ${ }_{2}$ and WAF(isoD/D)SAVAWR- $\mathrm{NH}_{2}$ were purified using gradients of $15-20 \% \mathrm{~B}$ in $23 \mathrm{~min}$ and $15-25 \%$ in $50 \mathrm{~min}$, respectively, on a $10 \mathrm{~mm}$ i.d. $\times 250 \mathrm{~mm} \mathrm{C18}$ column (Vydac, Hesperia, CA) at $4 \mathrm{~mL} / \mathrm{min}$ using Waters 510 HPLC pumps with gradient controller and model 490 wavelength detector (Millipore, Billerica, MA). Peptide sequences were confirmed by FTMS-ECD analysis. Stock solutions ranging from $0-100 \%$ isoAsp (10\% increments) for each set of isomeric peptides were prepared and diluted for FTMS-ECD analysis.

\section{Protein Digestion and Aging}

Cytochrome $c$ was digested with trypsin (100:1, wt/wt) in $0.1 \mathrm{M}$ ammonium bicarbonate for $5 \mathrm{~h}$ at $37^{\circ} \mathrm{C}$. The digest was dried to remove the buffer, $5 \%$ ammonium hydroxide ( $\mathrm{pH} 12$ ) was added, and the peptide solution was incubated overnight at $80^{\circ} \mathrm{C}$ to completely deamidate N31 of ${ }_{28}$ TGPNLHGLFGR $^{38}$ to the isoAsp/ Asp mixture. The solution was dried under vacuum centrifugation to remove ammonia and then analyzed by FTMS-ECD as described below. The peptide ${ }_{28}$ TGPDLHGLFGR $^{38}$ and its isoAsp counterpart from cytochrome $c$ used as calibration samples were previously $^{\circ}$ separated $^{\circ}[20]$.

\section{Mass Spectrometry}

ECD experiments were performed on a custom-built FTMS ${ }^{\circ}$ instrument ${ }^{\circ}$ previously ${ }^{\circ}$ described $[44,45]$. Peptide solutions were prepared at $\sim 10^{-5} \mathrm{M}$ from stock solutions in a 49.5:49.5:1 water:methanol:formic acid solution and $5 \mu \mathrm{L}$ was loaded into a glass capillary with 1 $\mu \mathrm{m}$ orifice made using mechanical tip puller (Sutter Instruments, Novato, CA). Applying $\sim 1.2 \mathrm{kV}$ to the solution generated positively charged ions used for FTMS-ECD analysis. For each experiment, the $(\mathrm{M}+$ $2 \mathrm{H})^{2+}$ species was isolated by Q1, accumulated in Q2 for 100-1000 ms, transferred to the ICR cell by gated trapping, and then irradiated with electrons at $0.25 \mathrm{eV}$ for 50-200 ms. ECD fragments were detected using a typical excitation/detection sequence with 20 scan signal averaging. Accumulation times for $(\mathrm{M}+2 \mathrm{H})^{2+}$ were optimized to achieve a signal/noise ratio of $\sim 500$. All analyses were performed in triplicate to generate some understanding of abundance variance upon ECD.

\section{Results and Discussion}

ECD of all isoAsp peptides generated the $z_{n}-57$ diagnostic fragment ion that was not found in corresponding Asp ECD spectra (e.g., YWQHTADQFR- $\mathrm{NH}_{2},{ }^{\circ}$ Figure $\left.^{\circ} 2 \mathrm{a}\right) .^{\circ}$ All $^{\circ}$ mixtures $^{\circ}$ showed $^{\circ}$ increasing $^{\circ} \mathrm{z}_{\mathrm{n}}-57$ abundance with increasing isoAsp content. For exam- 


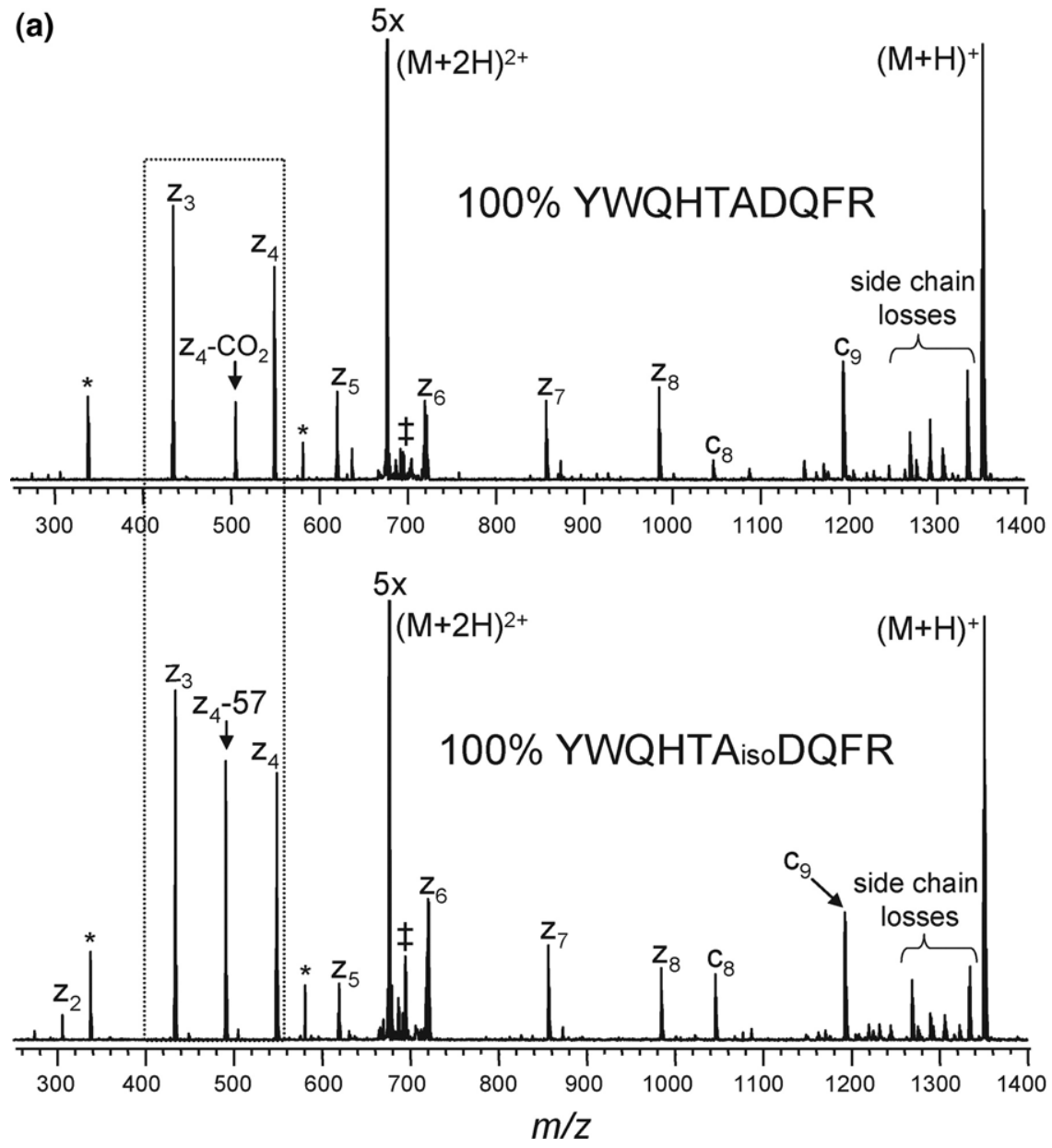

(b)
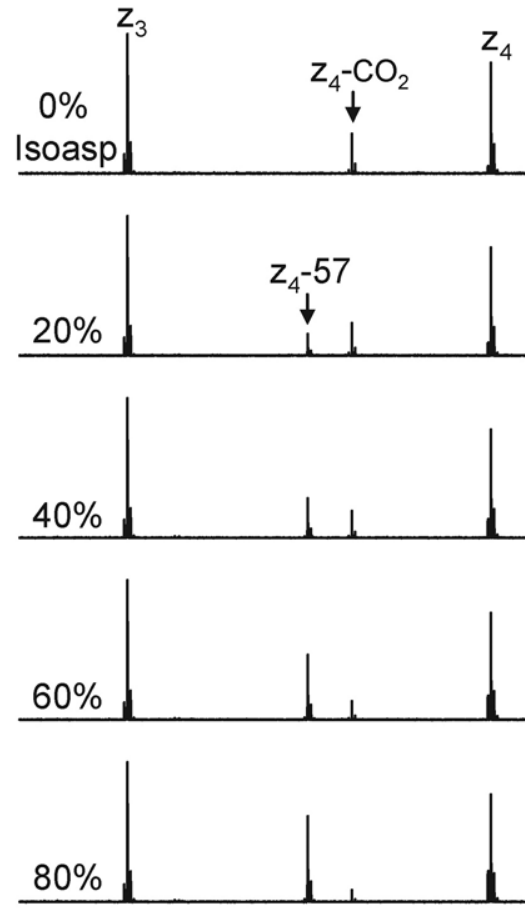

Figure 2. (a) ECD fragment spectra of YWQHTADQFR-NH $\mathrm{N}_{2}$ and $Y W Q H T A i s o D Q F R-\mathrm{NH}_{2}$. Double daggers represent ions from incomplete isolation and asterisks represent electronic noise or harmonics. (b) The 400-560 m/z region from the ECD fragment spectra of YWQHTA(D/isoD)QFR-NH ${ }_{2}$ mixtures [boxed region from (a)].

ple, $^{\circ}$ Figure $^{\circ} 2 \mathrm{~b}^{\circ}$ shows $^{\circ}$ the ${ }^{\circ} \mathrm{z}_{7}-57$ ion from YWQHTADQFR- $\mathrm{NH}_{2}$ at different Asp/isoAsp ratios, clearly demonstrating the changes in relative abundance of this ion. Relative abundance of the $z_{n}-57$ fragment $\left(\operatorname{Rel}\left[z_{n}-57\right]\right)$ with respect to all backbone fragments found in the spectra $(y, z, c$, and their neutral losses) was calculated and plotted against \% isoAsp content ${ }^{\circ}$ for $^{\circ}$ all $^{\circ}$ peptide $^{\circ}$ mixtures $^{\circ}\left(\right.$ Figure $^{\circ} 3$ ).$^{\circ}$ All ${ }^{\circ}$ plots exhibited a near linear relationship with $R^{2}$ values greater than 0.98 but with different slopes.

For all three peptide mixtures, particular backbone fragment ion abundances change when the Asp or isoAsp residues are interchanged. The increasing or decreasing fraction of gas-phase structures belonging to one form versus another may account for these trends since intramolecular hydrogen bonding has been shown ${ }^{\circ}$ to $^{\circ}$ remain $^{\circ}$ after $^{\circ} \mathrm{ECD}^{\circ}$ cleavage $^{\circ}\left[46,{ }^{\circ} 47\right] .{ }^{\circ}$ For $^{\circ}$ the peptide WAFDSAVAWR- $\mathrm{NH}_{2}$, no fragments that account for at least $5 \%$ of the total fragment abundance (excluding cleavage on either side of isoAsp/Asp) change more than $50 \%$, suggesting only a minor change in gas-phase structure with isoAsp substitution. How-

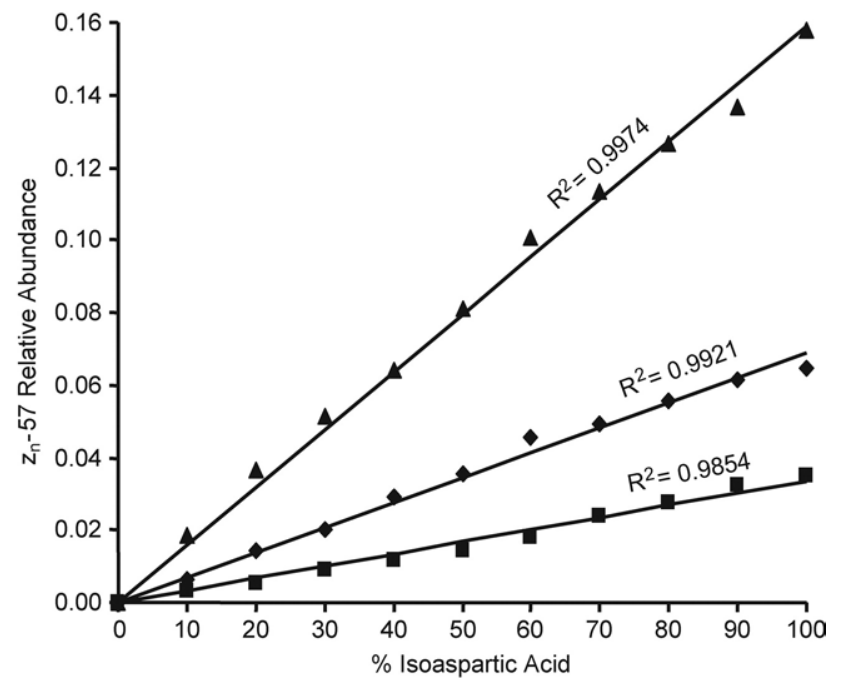

Figure 3. Plots of the $z_{n}-57$ relative abundance versus \% isoAsp content for; [filled triangle], YWQHTADQFR- $\mathrm{NH}_{2}$; [filled diamond], WAFDSAVAWR- $\mathrm{NH}_{2}$; [filled square], YDFIEYVR- $\mathrm{NH}_{2}$. 

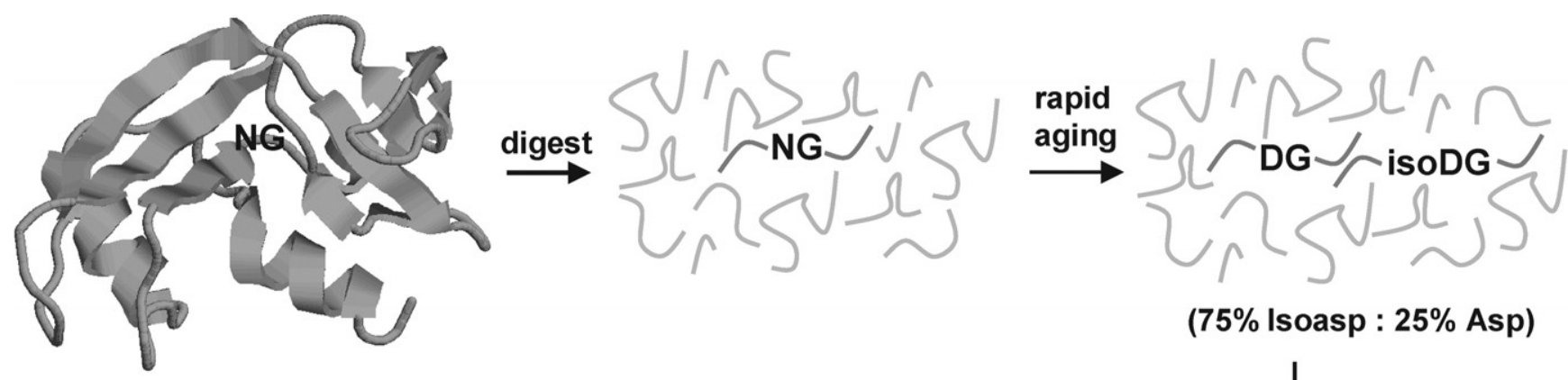

(75\% Isoasp : $25 \%$ Asp)

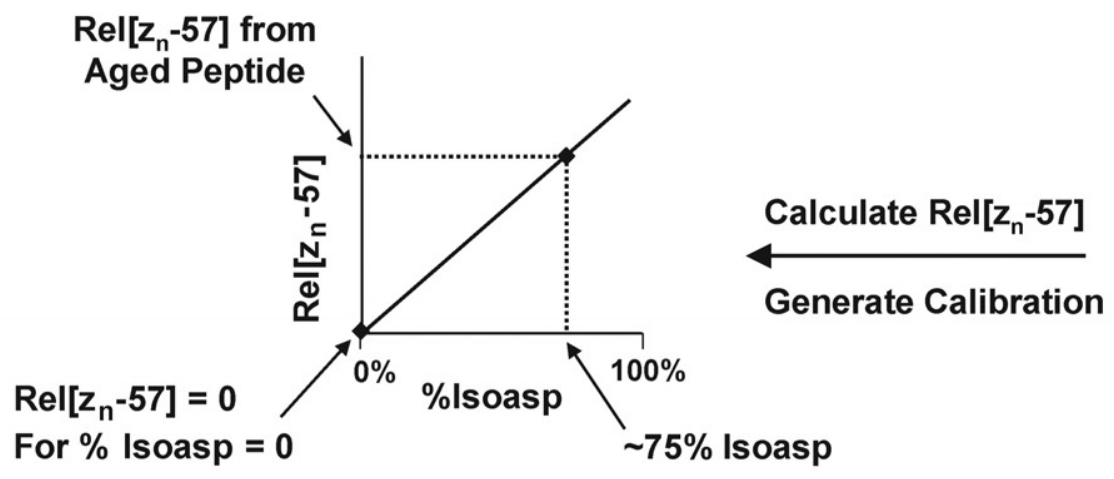

isolation/ECD of $\sim$ (isoD/D)G

Figure 4. Scheme for generating a two-point calibration from a deamidated peptide from a protein digest.

ever, the same comparison for YWQHTADQFR-NH $\mathrm{N}_{2}$ and YDFIEYVR- $\mathrm{NH}_{2}$ show 60 and $33 \%$ of the fragment abundances experience a $50 \%$ change or more with isoAsp substitution. This is probably due to the glutamic acid in $\mathrm{Y}(\mathrm{isoD} / \mathrm{D}) \mathrm{FIEYVR}-\mathrm{NH}_{2}$ and two glutamines and histidine residues in YWQHTA(isoD/ D)QFR-NH ${ }_{2}$. Hydrogen bonding involving these residues may be hindered or favored when interchanging Asp and isoAsp residues. Therefore, determining \% isoAsp content using the relative intensities of $z_{n}-57$ and any other particular backbone fragment that unknowingly varies may generate erroneous results. Despite the fluctuations in ion abundances, the data demonstrates the trend found between $\operatorname{Rel}\left[\mathrm{z}_{\mathrm{n}}-57\right]$ and \% isoAsp content is linear indicating that total fragment ion abundance remains fairly constant even if particular ions show substantial abundance variation. Furthermore, the linearity of these plots appears to be sequence independent but that relationship itself (the slope) is peptide dependent. Similar trends may be observed for CAD spectra since fragments resulting from cleavages adjacent to Asp residues change in abundance upon isoAsp ${ }^{\circ}$ substitution ${ }^{\circ}$ [32-35]. ${ }^{\circ}$ However, ${ }^{\circ}$ detection ${ }^{\circ}$ alone by CAD relies on discerning the relative intensities of fragments whose formation are collision energy dependent, so careful control over experimental parameters are probably necessary to generate useful calibration curves. The isoAsp diagnostic ion from ECD spectra offers unambiguous detection and calibration plots that pass through the origin offering a more straightforward procedure over CAD. Despite the advantage of using ECD, relative quantitation of asparaginyl deamidation or Asp isomerization products by either technique requires synthetic peptides for generating calibration curves.

Since random coil peptides typically produce a 3:1 ratio of isoAsp:Asp upon asparagine deamidation, the enzymatic digest peptide from the protein itself can potentially be used to generate a standard for quantitation. ${ }^{\circ} \mathrm{Clarke}^{\circ}[15],{ }^{\circ}$ Aswad ${ }^{\circ}[16],{ }^{\circ} \mathrm{Capasso}^{\circ}[17]^{\circ}$ and $^{\circ}$ others [18-20 $]^{\circ}$ have $^{\circ}$ shown $^{\circ}$ asparagine ${ }^{\circ}$ deamidation ${ }^{\circ}$ in ${ }^{\circ}$ linear peptides to produce mixtures ranging from 70 to $80 \%$ in favor of the isoAsp form. Considering these data, rapid aging of a peptide with an asparaginyl residue released from a protein by enzyme digestion should produce the $\sim 75 \%$ isoAsp mixture that can be used for calibration. A two point calibration line generated connecting the $\operatorname{Rel}\left[z_{n}-57\right]$ (75\% point) from the peptide ECD spectrum and the point of origin can then be used to quantify the relative abundance of products from the in vivo or in vitro $^{\circ}$ deamidation ${ }^{\circ}$ of $^{\circ}$ the $^{\circ}$ protein $^{\circ}\left(\right.$ Figure $\left.^{\circ} 4\right)$.

Cytochrome ${ }^{\circ} \mathrm{C}$ was $^{\circ}$ chosen $^{\circ}$ to $^{\circ}$ test $^{\circ}$ this $^{\circ}$ method $^{\circ}$ in $^{\circ} \mathrm{a}$ real protein. The tryptic peptide, ${ }_{28}$ TGPNLHGLFGR ${ }^{38}$ was previously deamidated and its isoforms were separated ${ }^{\circ}$ by $^{\circ} \mathrm{HPLC}[20] .{ }^{\circ} \mathrm{A}^{\circ}$ two-point ${ }^{\circ}$ calibration ${ }^{\circ}$ equation therefore already exists $(\mathrm{y}=0.0259 \mathrm{x})$ using the $\mathrm{ECD}$ spectra of the $100 \%$ Asp and $100 \%$ isoAsp peptide, and was used to test whether a $\sim 75 \%$ isoAsp mixture is generated $^{\circ}$ by $^{\circ}$ this $^{\circ}$ method $^{\circ}\left(\right.$ Figure $^{\circ}$ 5). ${ }^{\circ} \mathrm{A}^{\circ} 5$-h ${ }^{\circ}$ trypsin digestion of cytochrome $c$ produced the peptide 
${ }^{28} \mathrm{TGP}$ (isoD/D)LHGLFGR 38
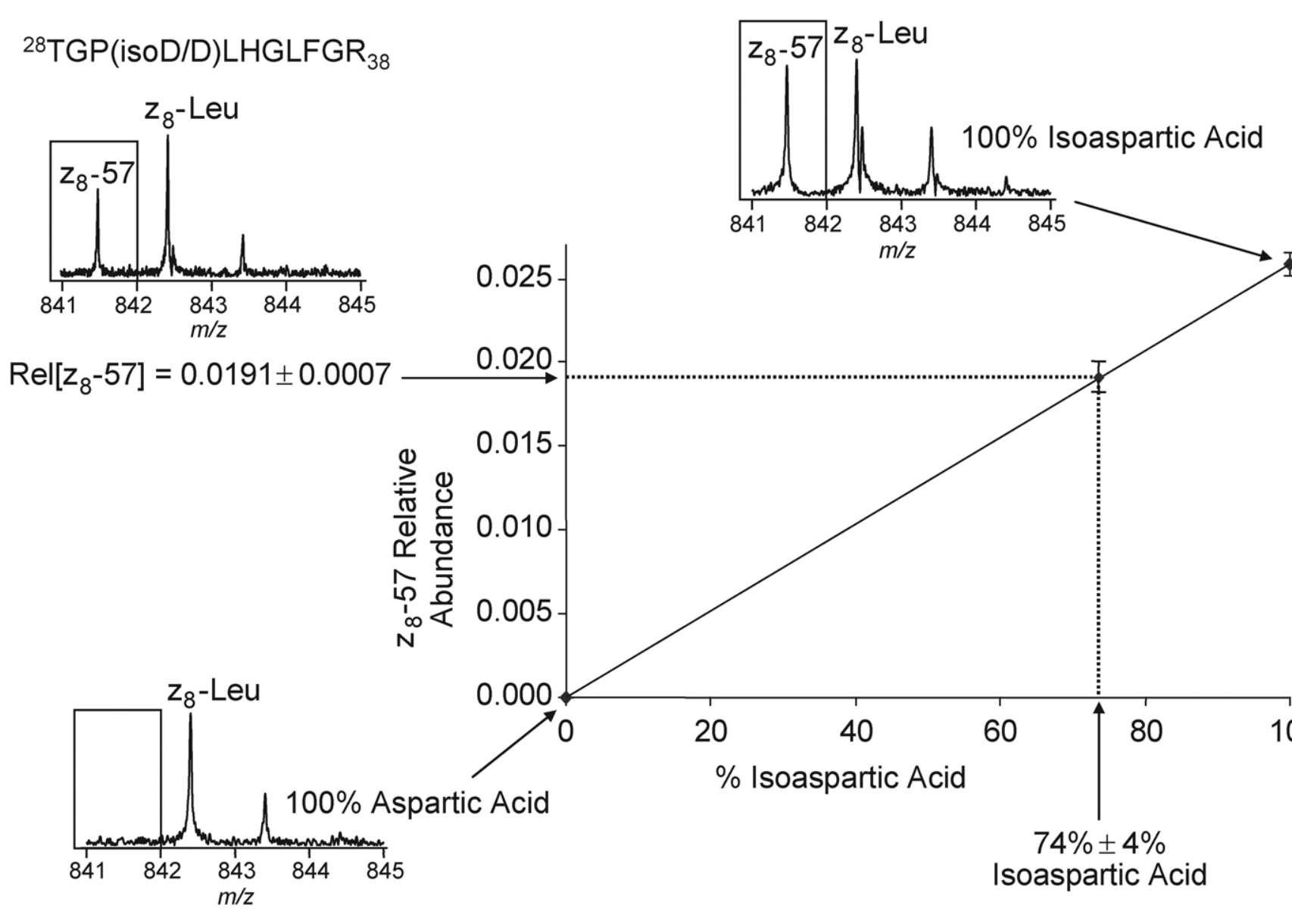

Figure 5. Rel[ $\left[z_{8}-57\right]$ from the ECD spectra of deamidated TGPNLHGLFGR corresponds to $74 \% \pm 4 \%$ using the plot of \% isoAsp content versus Rel[ $\left.z_{8}-57\right]$ constructed from the ECD fragment spectra of TGPDLHGLFGR and TGPisoDLHGLFGR, previously separated.

${ }_{28}$ TGPNLHGLFGR $^{38}(\mathrm{~m} / \mathrm{z}=584.815,2+)$ that was to be deamidated. Leucine on the C-terminal side of asparagine is believed to sterically hinder deamidation $(1 / 2$ life ${ }^{\circ} \sim{ }^{\circ} 128^{\circ}$ days $^{\circ}$ at ${ }^{\circ} \mathrm{pH} 7.4^{\circ}$ and $\left.{ }^{\circ} 37^{\circ} \mathrm{C}\right)^{\circ}[10]^{\circ}$ so harsh aging conditions were used to deamidate the peptide. FTMS analysis of the tryptic digest after a $16 \mathrm{~h}$ incubation at $\mathrm{pH} 12$ and $80^{\circ} \mathrm{C}$ showed complete deamidation indicated by a +0.984 Da shift in its isotopic pattern $(\mathrm{m} / \mathrm{z}=$ $585.307,2+)$. The ECD spectrum of the peptide confirmed the sequence was a mixture as revealed by presence of the $z_{8}-57$ fragment ion and a peak corresponding to the loss of an Asp side-chain ([M + $\left.2 \mathrm{H}]^{+\cdot}-60 \mathrm{Da}\right)^{\circ}[37]^{\circ} .^{\circ} \mathrm{A}^{\circ}$ value $^{\circ}$ of ${ }^{\circ} 0.0191^{\circ} \pm{ }^{\circ} 0.0007^{\circ}$ for ${ }^{\circ}$ the $\operatorname{Rel}\left[\mathrm{z}_{8}-57\right]$ calculated from the ECD spectra corresponds to $74 \% \pm 4 \%$ isoAsp content which falls near the expected ${ }^{\circ} 75 \%{ }^{\circ}$ value $^{\circ}$ (Figure $\left.{ }^{\circ} 5\right) .{ }^{\circ}$ This $^{\circ}$ supports $^{\circ}$ the ${ }^{\circ}$ hypothesis from the procedure presented that a two-point calibration based on the $\sim 75 \%$ point ( $\%$ unknown isoD $=\operatorname{Rel}[$ isoD $($ unknown $)] / \operatorname{Rel}[$ isoD $(75 \%$ point $)] \times 75 \%)$ can be used to determine the relative abundance of deamidation products from a protein without the need for synthetic peptides.

Determining the relative abundance of isoAsp residues resulting from Asp isomerization using the proce- dure may be possible, but there is no mass change with isomerization, and the point at which the ratio remains constant represents complete Asp isomerization. Peptides containing the DG sequence were shown to produce a 3:1 (isoAsp:Asp) ratio at equilibrium when incubated ${ }^{\circ}{ }^{\circ}{ }^{\circ}$ basic ${ }^{\circ}$ environment ${ }^{\circ}\left(\mathrm{pH}^{\circ}>7\right)$ [17], 'suggesting that the method described above could be applied to Asp isomerization. However, the rate of Asp isomerization is about 40 times slower than'deamidation [2], so longer and harsher conditions than those typically used for deamidation may be required to force complete isomerization, and such conditions may affect the 3:1 predicted ratio. For example, direct hydrolysis of the side chain amide group to a carboxyl group is promoted when'succinimideformation is hindered'([1],p.32). This situation would shift the ratio of products away from the expected 3:1 ratio and even more so if the Cterminal neighbor to Asp is a bulky residue. Therefore, additional data may be necessary to confidently say the method proposed above is applicable to Asp isomerization and synthetic standards may be necessary for quantitating the ratio.

All peptides for this study were synthesized with C-terminal arginines to simulate tryptic peptides, and 
their sequences were varied in number of polar/ nonpolar residues and position of the isoAsp residue to better represent the sequence diversity found in proteins. The reproducibility of the linear relationship among the four peptides shown strengthens the claim that this method is a useful tool for relative isoAsp/Asp quantitation from asparagine deamidation but only when trypsin is used for protein digestion. The sequence diversity of peptides generated using other enzymes (e.g., Glu-C, Lys-C) should statistically be similar to trypsin although their size may be larger. This suggests the applicability of using the isoaspartyl quantitation method to those employing an enzyme other than trypsin, although additional experiments should be conducted to test this claim.

The procedure for isoaspartyl quantitation by ECD assumes that a peptide, when harshly deamidated with high temperature, will always achieve a 3:1 ratio. While this assumption is clearly true in the case studied here, it may not be true for all peptides. For example, the competing pathway of direct hydrolysis could alter the ratio when C-terminal neighbors to asparagine are those that greatly reduce the rate of deamidation (e.g., valine, ${ }^{\circ}$ isoleucine $)^{\circ}\left([1],{ }^{\circ}\right.$ p. $\left.{ }^{\circ} 32\right) .{ }^{\circ}$ Also, ${ }^{\circ} \mathrm{Asp}^{\circ}$ and ${ }^{\circ}$ isoAsp could reform the succinimide intermediate when longer incubation periods are required for complete deamidation. Therefore, in cases where more accurate numbers are needed, full calibration plots will have to be generated using pure isoAsp and Asp peptides either from HPLC fractionation, as shown with cytochrome $c$, or synthetically produced, peptides as shown above. Nevertheless, the 3:1 mol ratio from the method presented does generate a good starting point and accurate relative estimates.

Finally, to successfully quantitate isoaspartyl residues, a suitable peptide must be obtained that contains the deamidation site and that generates an ECD spectrum with the isoaspartyl diagnostic ion. Some practical obstacles therefore may be encountered when employing the method such as procuring enough protein for the procedure, isolating a peptide with the deamidation site in the mass spectrometer, and generating a useful ECD spectrum from that peptide for quantitation. First, the low-level amount of proteins typically obtained from biological samples is a practical limit to this and most $^{\circ}$ quantitation ${ }^{\circ}$ methods ${ }^{\circ}$ and ${ }^{\circ}$ may $^{\circ}$ prohibit $^{\circ}$ the ${ }^{\circ}$ use ${ }^{\circ}$ of the method entirely or require extensive chromatographic cleanup procedures. Other methods may be necessary, ${ }^{\circ}$ such $^{\circ}$ as $^{\circ}$ immunological $^{\circ}[48]^{\circ}$ or $^{\circ}$ radioactive [25] $]^{\circ}$ methods, ${ }^{\text {but }}{ }^{\circ}$ these ${ }^{\circ}$ methods ${ }^{\circ} \mathrm{can}^{\circ}$ only ${ }^{\circ}$ detect ${ }^{\circ}$ isoaspartyl residues, and cannot localize or quantitate the relative abundance of deamidation products. Second, as proteins become larger, their digestion mixtures become more complex. This may result in overlapping species in the broadband ESI spectrum, making isolation of the peptide of interest difficult or impossible. Fractionation of the digest therefore may be necessary to reduce the complexity of the mixture, but not as rigorous a separation as one required to differentiate isoAsp- from Asp-containing peptides. Furthermore, the peptides used in the experiments were relatively small $(<\sim 1500 \mathrm{Da})$ and the ECD spectrum of the $2^{+}$ charge state for each peptide fortunately showed all interresidue cleavages including the isoaspartyl diagnostic ion. Therefore, it is important to use a peptide that is favorable for ECD analysis. There are several factors that affect the ECD process, such as the increase in $^{\circ}$ capture ${ }^{\circ}$ cross-section ${ }^{\circ}$ with ${ }^{\circ}$ charge $[49]$, the ${ }^{\circ}$ hydrogen bonding between fragments that remain after the ECD event $[46,47]$, and the ${ }^{\circ}$ distance ${ }^{\circ}$ between ${ }^{\circ} e^{\circ}$ charges and cleavage ${ }^{\circ}$ sites $^{\circ}[50]^{\circ}{ }^{\circ}$ Because ${ }^{\circ}$ of ${ }^{\circ}$ these ${ }^{\circ}$ factors, ${ }^{\circ} a^{\circ}$ multiply protonated peptide whose charges are uniformly distributed is preferred to facilitate a complete cleavage pattern and ensure detection of a possible isoaspartyl residue, but it is not always necessary. For example, ECD of the $3^{+}$charge state of a $\sim 2850$ Da tryptic fragment from ribonuclease A showed the diagnostic isoaspartyl fragment ion but failed to produce fragments ${ }^{\circ}$ representing ${ }^{\circ}$ all ${ }^{\circ}$ interresidue ${ }^{\circ}$ cleavages $^{\circ}[20] .{ }^{\circ}$ Despite all the possible hurdles discussed, the procedure presented should provide a convenient, MS-based method for relative isoaspartyl quantitation although additional steps may be necessary to obtain the necessary peptide and generate an adequate ECD spectrum for calculating $\operatorname{Rel}\left[z_{n}-57\right]$.

\section{Conclusions}

The plots of $\operatorname{Rel}\left[z_{n}-57\right]$ versus \% isoAsp content for three sets of synthetic peptides suggest that the linear relationship found is peptide independent but that the slopes are sequence-dependent, and synthetic standards of each form (isoAsp and Asp) are required for generating calibration curves. However, a method is suggested for determining the relative amounts of protein deamidation products from in vivo or in vitro experiments without the need to synthetic standards. The procedure involves rapid and harsh aging of the proteolytic peptide containing the deamidation site that can be used to generate a standard mixture for calibration, assuming that the often observed 3:1 (isoAsp:Asp) molar ratio holds true for the peptide of interest, for determining the relative amounts of protein deamidation products. This assumption was tested for cytochrome $c$ and appears to be valid.

Two important attributes of using ECD to quantitate the relative abundance of isoAsp residues are the elimination of both the need for HPLC separation and the necessity of peptide standards for relative quantitation. Elimination of the HPLC step is beneficial because it dramatically shortens experiment time and decreases sample consumption. This is important considering the amount of protein obtained from typical proteomic experiments. Peptide synthesizers remove tedious manual peptide synthesis but steps such as cleanup procedures and peptide characterization are still required, so 
obtaining the standard directly from the protein is not only facile but also economical.

In conclusion, the method presented should be a practical approach for studies involving proteins that have been determined to deamidate but for which the relative abundances of their Asp and isoAsp products are still unknown.

\section{Acknowledgments}

The authors thank Bruce Thomson of MDS Sciex for instrument spare parts, Dr. Arthur Robinson for helpful discussions, NIH/ NCRR P41RR10888, NIH/NHLBI N01HV28178, MDS Sciex, and the American Petroleum Research Fund for financial support.

\section{References}

1. Robinson, A. B.; Robinson, N. E. Molecular Clocks: Deamidation of Asparaginyl and Glutaminyl Residues in Peptides and Proteins; Althouse Press: Cave Junction, OR, 2004.

2. Clarke, S. Propensity for Spontaneous Succinimide Formation from Aspartyl and Asparaginyl Residues in Cellular Proteins. Int. J. Pept. Protein Res. 1987, 30, 808-821.

3. Aswad, D. W.; Paranandi, M. V.; Schurter, B. T. Isoaspartate in Peptides and Proteins: Formation, Significance, and Analysis. J. Pharm. Biomed. Anal. 2000, 21, 1129-1136.

4. Hanson, S. R. A.; Hasan, A.; Smith, D. L.; Smith, J. B. The Major in Vivo Modifications of the Human Water-Insoluble lens Crystallins are Disulfide Bonds, Deamidation, Methionine Oxidation, and Backbone Cleavage. Exp. Eye Res. 2000, 71, 195-207.

5. Nilsson, M. R.; Driscoll, M.; Raleigh, D. P. Low Levels of Asparagine Deamidation Can Have a Dramatic Effect on Aggregation of Amyloidogenic Peptides: Implications for the Study of Amyloid Formation. Protein Sci. 2002, 11, 342-349.

6. Ribot, W. J.; Powell, B. S.; Ivins, B. E.; Little, S. F.; Johnson, W. M.; Hoover, T. A.; Norris, S. L. Adamovicz, J. J.; Friedlander, A. M. Andrews, G. P. Comparative Vaccine Efficacy of Different Isoforms of Recombinant Protective Antigen Against Bacillus anthracis Spore Challenge in Rabbits. Vaccine 2006, 24, 3469-3476.

7. Gershon, H.; Gershon, D. Inactive Enzyme Molecules in Aging Mice: Liver Aldolase. Proc. Natl. Acad. Sci. U.S.A. 1973, 70, 909-913.

8. Deverman, B. E.; Cook, B. L.; Manson, S. R.; Niederhoff, R. A.; Langer, E. M.; Rosova, I.; Kulans, L. A.; Fu, X. Y.; Weinberg, J. S.; Heinecke, J. W.; Roth, K. A.; Weintraub, S. J. Bcl-X-L Deamidation is a Critical Switch in the Regulation of the Response to DNA Damage. Cell 2002, $111,51-62$.

9. Friedman, A. R.; Ichhpurani, A. K.; Brown, D. M.; Hillman, R. M.; Krabill, L. F.; Martin, R. A.; Zurcherneely, H. A.; Guido, D. M. Degradation of Growth-Hormone Releasing-Factor Analogs in Neutral Aqueous-Solution Is Related to Deamidation of Asparagine ResiduesReplacement of Asparagine Residues by Serine Stabilizes. Int. J. Pept. Protein Res. 1991, 37, 14-20.

10. Robinson, N. E.; Robinson, A. B. Molecular Clocks. Proc. Natl. Acad. Sci. U.S.A. 2001, 98, 944-949.

11. Robinson, N. E.; Robinson, A. B. Prediction of Protein Deamidation Rates from Primary and Three-Dimensional Structure. Proc. Natl. Acad. Sci. U.S.A. 2001, 98, 4367-4372.

12. Hellman, U.; Wernstedt, C.; Gonez, J.; Heldin, C. H. Improvement of an In-Gel Digestion Procedure for the Micropreparation of Internal Protein-Fragments for Amino-Acid Sequencing. Anal. Biochem. 1995, 224 , $451-455$.

13. Krokhin, O. V., Antonovici, M., Ens, W., Wilkins, J. A., Standing, K. G. Deamidation of -Asn-Gly- Sequences during Sample Preparation for Proteomics: Consequences for MALDI and HPLC-MALDI Analysis. Anal. Chem. 2006, Web Release July 21, 2006.

14. Hsu, Y. R.; Chang, W. C.; Mendiaz, E. A.; Hara, S.; Chow, D. T.; Mann, M. B.; Langley, K. E.; Lu, H. S. Selective Deamidation of Recombinant Human Stem Cell Factor During in Vitro Aging; Isolation and Characterization of the Aspartyl and Isoaspartyl Homodimers and Heterodimers. Biochemistry 1998, 37, 2251-2262.

15. Geiger, T.; Clarke, S. Deamidation, Isomerization, and Racemization at Asparaginyl and Aspartyl Residues in Peptides. J. Biol. Chem. 1987, 262, 785-795

16. Potter, S. M.; Henzel, W. J.; Aswad, D. W. In Vitro Aging of Calmodulin Generates Isoaspartate at Multiple Asn-Gly and Asp-Gly Sites in Calcium-Binding Domain-Ii, Domain-Iii, and Domain-Iv. Protein Sci. 1993, 2, 1648-1663.

17. Capasso, S. Thermodynamic Parameters of the Reversible Isomerization of Aspartic Residues via a Succinimide Derivative. Thermochim. Acto 1996, 286, 41-50.
18. Meinwald, Y. C.; Stimson, E. R.; Scheraga, H. A. Deamidation of the Asparaginyl-Glycyl Sequence. Int. J. Pept. Protein Res. 1986, 28 $79-84$.

19. Chelius, D.; Rehder, D. S.; Bondarenko, P. V. Identification and Characterization of Deamidation Sites in the Conserved Regions of Human Immunoglobulin $\gamma$ Anitbodies. Anal. Chem. 2005, 77, 6004-6011.

20. Cournoyer, J. J.; Lin, C.; O'Connor, P. B. Detecting Deamidation Products in Proteins by Electron Capture Dissociation. Anal. Chem. 2006, $78,1264-1271$.

21. Tyler-Cross, R.; Schirch, V. Effects of Amino Acid Sequence, Buffers, and Ionic Strength on the Rate and Mechanism of Deamidation of Asparagine Residues in Small Peptides. J. Biol. Chem. 1991, 266, 22549 22556.

22. Athiner, L.; Kindrachuk, J.; Georges, F.; Napper, S. The Influence of Protein Structure on the Products Emerging from Succinimide Hydrolysis. J. Biol. Chem. 2002, 277, 30502-30507.

23. Capasso, S.; Di Cerbo, P. Kinetic and Thermodynamic Control of the Relative Yield of Deamidation of Asparagine and Isomerization of Aspartic Acid Residues. J. Peptide Res. 2000, 56, 382-387.

24. Cirrito, T. P.; Pu, Z; Deck, M. B.; Unanue, E. R. Deamidation of Asparagine in a Major Histocompatibility Complex-Bound Peptide Affects T Cell Recognition but Does Not Explain Type B Reactivity. J. Exp. Med. 2001, 194, 1165-1170

25. Carslon, A. D.; Riggin, R. M. Development of Improved High-Performance Liquid Chromatography Conditions for Nonisotopic Detection of Isoaspartic Acid to Determine the Extent of Protein Deamidation. Anal. Biochem. 2000, 278, 150-155.

26. Huang, L. H.; Li, J. R.; Wroblewski, V. J.; Beals, J. M.; Riggin, R. M. In Vivo Deamidation Characterization of Monoclonal Antibody by LC/ MS/MS. Anal. Chem. 2005, 77, 1432-1439.

27. Di Donato, A.; Ciardiello, M. A.; Denigris, M.; Piccoli, R.; Mazzarella, L.; Dalessio, G. Selective Deamidation of Ribonuclease-A Isolation and Characterization of the Resulting Isoaspartyl and Aspartyl Derivatives. J. Biol. Chem. 1993, 268, 4745-4751.

28. Solstad, T.; Carvalho, R. N.; Andersen, O. A.; Waidelich, D.; Flatmark, T. Deamidation of Labile Asparagine Residues in the Autoregulatory Sequence of Human Phenylalanine Hydroxylase-Structural and Functional Implications. Eur. J. Biochem. 2003, 270, 929-938.

29. Smyth, D. G.; Stein, W. H.; Moore, S. On the Sequence of Residues 11 to 18 in Bovine Pancreatic Ribonuclease. J. Biol. Chem. 1962, 237, 1845-1850.

30. McLafferty, F. W. Tandem Mass Spectrometry; Wiley: New York, 1983.

31. Shukla, A. K.; Futrell, J. H. Collisional Activation and Dissociation of Polyatomic Ions. Mass Spectrom. Rev. 1993, 12, 211-255.

32. Lehmann, W.; Schlosser, A.; Erben, G.; Pipkorn, R.; Bossemeyer, D. Kinzel, V. Analysis of Isoaspartate in Peptides by Electrospray Tandem Mass Spectrometry. Protein Sci. 2000, 9, 2268-2290.

33. Gonzalez, L. J.; Shimizu, T.; Satomi, Y.; Betancourt, L.; Besada, V.; Padron, G.; Orlando, R.; Shirasawa, T.; Shimonishi, Y.; Takao, T. Differentiating $\alpha$ - and $\beta$-Aspartic Acids by Electrospray Ionization and Low-Energy Tandem Mass Spectrometry. Rapid Commun. Mass Spectrom. 2000, 14, 2092-2102.

34. Schindler, P.; Muller, D.; Marki, W.; Grossenbacher, H.; Richter, W. J. Characterization of a $\beta$-Asp33 Isoform of Recombinant Hirudin Sequence Variant 1 by Low-Energy Collision-Induced Dissociation. J. Mass Spectrom. 1996, 31, 967-974.

35. Luu, N. C.; Robinson, S.; Zhao, R.; McKean, R.; Ridge, D. P.Mass Spectrometric Differentiation of $\alpha$ - and $\beta$-Aspartic Acid in a PseudoTetrapeptide Thrombosis Inhibitor and Its Isomer. Eur. J. Mass Spectrom. 2004, 10, 279-287.

36. Zubarev, R. A.; Kelleher, N. L.; McLafferty, F. W. Electron Capture Dissociation of Multiply Charged Protein Cations. A Nonergodic Process. J. Am. Chem. Soc. 1998, 120, 3265-3266.

37. Cournoyer, J. J.; Pittman, J. L.; Ivleva, V. I.; Fallows, E.; Waskell, L.; Costello, C. E.; O'Connor, P. B. Deamidation: Differentiation of Aspartyl from Isoaspartyl Products in Peptides by Electron Capture Dissociation. Protein Sci. 2005, 14, 452-463.

38. O'Connor, P. B.; Cournoyer, J. J.; Pitteri, S. J.; Chrisman, P. A. McLuckey, S. A. Differentiation of Aspartic and Isoaspartic Acids Using Electron Transfer Dissociation. J. Am. Soc. Mass Spectrom. 2006, 17 15-19.

39. Stensballe, A.; Jensen, O. N.; Olsen, J. V.; Haselmann, K. F.; Zubarev, R. A. Electron Capture Dissociation of Singly and Multiply Phosphorylated Peptides. Rapid Commun. Mass Spectrom. 2000, 14, 1793-1800.

40. Mirgorodskaya, E.; Roepstorff, P.; Zubarev, R. A. Localization of O-Glycosylation Sites in Peptides by Electron Capture Dissociation in a Fourier Transform Mass Spectrometer. Anal. Chem. 1999, 71, 4431-4436.

41. Cooper, H. J.; Hudgins, R. R.; Hakansson, K.; Marshall, A. G. Characterization of Amino Acid Side-Chain Losses in Electron Capture Dissociation. J. Am. Soc. Mass Spectrom. 2002, 13, 241-249.

42. Desaire, H.; Leary, J. A. Detection and Quantification of the Sulfated Disaccharides in Chondroitin Sulfate by Electrospray Tandem Mass Spectrometry. J. Am. Soc. Mass Spectrom. 2000, 11, 916-920.

43. Hitchcock, A. M.; Costello, C. E.; Zaia, J. Glycoform Quantification of Chondroitin/Dermatan Sulfate Using a Liquid ChromatographyTandem Mass Spectrometry Platform. Biochemistry 2006, 45, $2350-$ 2361. 
44. O'Connor, P. B.; Pittman, J. L.; Thomson, B. A.; Budnik, B. A.; Cournoyer, J. J.; Jebanathirajah, J.; Lin, C.; Moyer, S.; Zhao, C. A New Hybrid Electrospray Fourier Transform Mass Spectrometer: Design and Performance Characteristics. Rapid Commun. Mass Spectrom. 2006, 20, $259-266$.

45. Jebanathirajah, J. A.; Pittman, J. L.; Thomson, B. A.; Budnik, B. A.; Kaur, P.; Rape, M.; Kirschner, M.; Costello, C. E.; O'Connor, P. B. Characterization of a New qQq-FTICR Mass Spectrometer for Post-Translational Modification Analysis and Top-Down Tandem Mass Spectrometry of Whole Proteins. J. Am. Soc. Mass Spectrom. 2005, 16, 1985-1999.

46. Breuker, K.; Oh, H. B.; Horn, D. M.; Cerda, B. A.; McLafferty, F. W. Detailed Unfolding and Folding of Gaseous Ubiquitin Ions Characterized by Electron Capture Dissociation. J. Am. Chem. Soc. 2002, 124, 6407-6420.
47. Lin, C.; Cournoyer, J. C.; O'Connor, P. B. Use of a Double Resonance Electron Capture Dissociation to Probe Fragment Intermediate Lifetimes. J. Am. Soc. Mass Spectrom. 2006.

48. Shimizu, T.; Watanabe, A.; Ogawara, M.; Mori, H.; Shirasawa, T. Isoaspartate Formation and Neurodegeneration in Alzheimer's Disease. Arch. Biochem. Biophys. 2000, 381, 225-234.

49. Zubarev, R. A.; Horn, D. M.; Fridriksson, E. K.; Kelleher, N. L.; Kruger, N. A.; Lewis, M. A.; Carpenter, B. K.; McLafferty, F. W. Electron Capture Dissociation for Structural Characterization of Multiply Charged Protein Cations. Anal. Chem. 2000, 72, 563-573

50. Iavarone, A. T.; Paech, K.; Williams, E. R. Effects of Charge State and Cationizing Agent on the Electron Capture Dissociation of a Peptide. Anal. Chem. 2004, 76, 2231-2238. 